\title{
A comment on "Accurate spin axes and solar system dynamics: Climatic variations for the Earth and Mars"
}

\author{
J. Laskar \\ Astronomie et Systèmes Dynamiques, IMCCE-CNRS UMR 8028, 77 Av. Denfert-Rochereau, 75014 Paris, France \\ Received 19 November 2003 / Accepted 26 November 2003

\begin{abstract}
In a recent paper, Edvardsson et al. (2002) propose a new solution for the spin evolution of the Earth and Mars. Their results differ significantly with respect to previous studies, as they found a large contribution on the precession of the planet axis from the tidal effects of Phobos and Deimos. In fact, this probably results from the omission by the authors of the torques exerted on the satellites orbits by the planet's equatorial bulge, as otherwise the average torque exerted by the satellites on the planet is null.
\end{abstract}

Key words. celestial mechanics - ephemerides - planets and satellites: individual: Mars - Earth

\section{Introduction}

In a recent paper, Edvardsson et al. (2002) propose a new solution for the spin evolution of the Earth and Mars. Considering the absence of a precise evaluation of the errors due to the integrator and the absence of relativity in the model, one could discuss the use of the word "accurate" in the title of the paper, but unfortunately there are some more important flaws in this paper. In their integration of the spin of Mars, the authors found that the integration of the spin changes in a large amount when Phobos and Deimos are taken into account (see their Fig. 12). They also notice that their "curve without the moons is very similar to the curve given by Bouquillon \& Souchay (1999)", who included the moons. In fact, the authors have since realized that their paper is in error on this point.

\section{Precession due to a distant satellite}

Let us consider a planet $\mathcal{P}$ with momentum of inertia $A=B<C$ orbiting the Sun on a fixed ellipse (we will not consider here the planetary perturbations or the perturbations due to the satellite presence), and a satellite $\mathcal{S}$ of mass $m$ orbiting the planet. Let $(\boldsymbol{i}, \boldsymbol{j}, \boldsymbol{k})$ be a basis linked to $\mathcal{P}$ with $\boldsymbol{k}$ associated to the axis of maximum inertia $C$. Let $(\boldsymbol{I}, \boldsymbol{J}, \boldsymbol{K})$ be a fixed reference frame, with origin in the direction of $\boldsymbol{I}$, and $\boldsymbol{K}$ normal to the orbital plane of $\mathcal{P}$ (Fig. 1). $a$ and $e$ are the semi-major axis and eccentricity of $\mathcal{S}, i$ and $\Omega$ the inclination and longitude of the ascending node of the satellite orbit over the orbital plane of the planet $(\boldsymbol{I}, \boldsymbol{J})$, while $v$ is the true longitude, and $\omega$ the argument of perihelion. If $\boldsymbol{r}$ is the radius vector from the planet's

\footnotetext{
* e-mail: laskar@imcce.fr
}

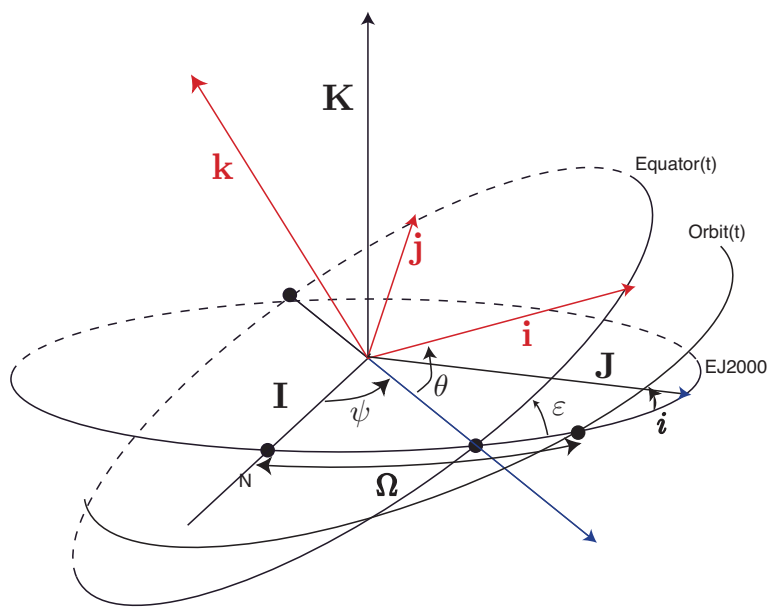

Fig. 1. The orbital plane is defined by the inclination $i$ and longitude of node $\Omega$ with respect to a fixed reference frame $(\boldsymbol{I}, \boldsymbol{J}, \boldsymbol{K})$, while $(\boldsymbol{i}, \boldsymbol{j}, \boldsymbol{k})$ are the axis of inertia of the planet.

to the satellite's center of mass, with modulus $r$ and unit vector $\boldsymbol{v}=\boldsymbol{r} / r$, the torque exerted by $\mathcal{S}$ on $\mathcal{P}$ is

$\Gamma=\frac{3 G m}{r^{3}} \boldsymbol{v} \wedge \mathcal{I} \boldsymbol{v}$

where $G$ is the gravitational constant and $\mathcal{I}$ the matrix of inertia $(\mathcal{I}=\operatorname{diag}(A, A, C))$. Noting that $\mathcal{I}=\operatorname{diag}(A, A, A)+$ $\operatorname{diag}(0,0, C-A),(1)$ can also be expressed as (see Murray 1983)

$\Gamma=-\frac{3 G m}{r^{3}}(C-A) \boldsymbol{k} \wedge\left(\boldsymbol{v} \boldsymbol{v}^{\tau}\right) \boldsymbol{k}$

where $\boldsymbol{v}^{\tau}$ denotes the transposed of $\boldsymbol{v}\left(\boldsymbol{v}^{\tau} \boldsymbol{k}\right.$ is thus the dot product of $\boldsymbol{v}$ and $\boldsymbol{k}$ ). We will average $\Gamma$ over the fastest angle of this 
problem, that is over the mean anomaly $M$ of the satellite (the rotational angle $\theta$ is removed after the assumption $A=B$ ). As $\boldsymbol{k}$ does not depend on $M$, the only expression to average in (2) is $\gamma=\boldsymbol{v} \boldsymbol{v}^{\tau} / r^{3}$. In the $(\boldsymbol{I}, \boldsymbol{J}, \boldsymbol{K})$ frame, the coordinates of $\boldsymbol{v}$ are

$\boldsymbol{v}=\mathcal{R}_{3}(\Omega) \mathcal{R}_{1}(i)\left(\begin{array}{c}\cos (v+\omega) \\ \sin (v+\omega) \\ 0\end{array}\right) \quad$ with

$\mathcal{R}_{1}(\alpha)=\left(\begin{array}{ccc}1 & 0 & 0 \\ 0 & \cos \alpha & -\sin \alpha \\ 0 & \sin \alpha & \cos \alpha\end{array}\right)$

$\mathcal{R}_{3}(\alpha)=\left(\begin{array}{ccc}\cos \alpha & -\sin \alpha & 0 \\ \sin \alpha & \cos \alpha & 0 \\ 0 & 0 & 1\end{array}\right)$.

As $r^{2} \mathrm{~d} v=a^{2} \sqrt{1-e^{2}} \mathrm{~d} M$, after averaging over $M$, we obtain

$<\gamma>_{M}=\frac{1}{2 a^{3}\left(1-e^{2}\right)^{3 / 2}}[I d-\mathcal{M}]$

where $\mathcal{M}$ is expressed in $(\boldsymbol{I}, \boldsymbol{J}, \boldsymbol{K})$ as the $3 \times 3$ matrix

$\mathcal{M}=\mathcal{R}_{3}(\Omega) \mathcal{R}_{1}(i)\left(\begin{array}{lll}0 & 0 & 0 \\ 0 & 0 & 0 \\ 0 & 0 & 1\end{array}\right) \mathcal{R}_{1}(-i) \mathcal{R}_{3}(-\Omega)$.

When the precession of the node is rapid with respect to the precession of the spin axis of the planet (as for the Moon around the Earth), one can also average over $\Omega$. We have thus in the basis $(\boldsymbol{I}, \boldsymbol{J}, \boldsymbol{K})$

$$
<\mathcal{M}>_{\Omega}=\frac{\sin ^{2} i}{2} I d+\left(1-\frac{3 \sin ^{2} i}{2}\right)\left(\begin{array}{lll}
0 & 0 & 0 \\
0 & 0 & 0 \\
0 & 0 & 1
\end{array}\right) .
$$

Finally, as the parts involving the identity Id cancel, we are left with

$$
\begin{aligned}
<\Gamma>_{M, \Omega} & =\frac{3 G m(C-A)}{2 a^{3}\left(1-e^{2}\right)^{3 / 2}}\left(1-\frac{3 \sin ^{2} i}{2}\right) \boldsymbol{k} \wedge \boldsymbol{K} \boldsymbol{K}^{\tau} \boldsymbol{k} \\
& =\frac{3 G m(C-A)}{2 a^{3}\left(1-e^{2}\right)^{3 / 2}}\left(1-\frac{3 \sin ^{2} i}{2}\right) \cos \varepsilon \boldsymbol{k} \wedge \boldsymbol{K}
\end{aligned}
$$

which leads to the classical contribution $p_{\mathrm{s}}$ of the precession of spin axis $\boldsymbol{k}$ around the normal to the orbital plane $\boldsymbol{K}$, when the rotational rate $v$ is large with respect to the precession frequency $p$.

$$
p_{s}=-\frac{3 G m}{2 v a^{3}\left(1-e^{2}\right)^{3 / 2}} \frac{C-A}{C}\left(1-\frac{3 \sin ^{2} i}{2}\right) \cos \varepsilon .
$$

\section{Precession due to a close satellite}

The previous study is in fact valid uniquely in the case of a satellite sufficiently far from the planet, so that the precession of its orbit is mostly driven by the solar perturbations, and not by the torque exerted by the equatorial bulge of the planet. In the case of a close satellite, it was shown (Goldreich 1965; Kinoshita 1993) that instead of precessing around the normal $\boldsymbol{K}$ to the orbital plane $(\boldsymbol{I}, \boldsymbol{J})$, with a roughly constant inclination $i$ with respect to $(\boldsymbol{I}, \boldsymbol{J})$, the satellite will precess with respect to $\boldsymbol{k}$ with a nearly constant inclination $i^{\prime}$ with respect to the equator plane $(\boldsymbol{i}, \boldsymbol{j})$. In this case, all the previous study is still valid, but the orbit of the satellite is now referred to the equatorial reference frame $(\boldsymbol{i}, \boldsymbol{j}, \boldsymbol{k})$, and the second averaging is made with respect to the longitude of the node, $\Omega^{\prime}$, of the satellite orbit with respect to the equatorial plane $(\boldsymbol{i}, \boldsymbol{j})$. The average expression (6), (7) is still valid, when replacing $\Omega$ and $i$ by $\Omega^{\prime}$ and $i^{\prime}$. The average $\left\langle\mathcal{M}>_{\Omega^{\prime}}\right.$ will then be given by (8), after changing $i$ with $i^{\prime}$, but this expression is now obtained in the reference frame of the planet $(\boldsymbol{i}, \boldsymbol{j}, \boldsymbol{k})$. As $\boldsymbol{k} \wedge \boldsymbol{k} \boldsymbol{k}^{\tau} \boldsymbol{k}=\boldsymbol{k} \wedge \boldsymbol{k}=0$, we have now for the averaged torque

$<\Gamma>_{M, \Omega^{\prime}}=0$.

\section{Discussion}

Thus, contrarily to what is found by Edvardsson et al. (2002), the averaged torque exerted by Phobos and Deimos on Mars should be null (at first order). The origin of the authors' error can be traced by looking to their Figs. 1 and 2. The inclination of the satellites with respect to the equator starts with a value close to zero at the origin, and then increases up to about 50 degrees and then back to zero with a period of about 6000 and 1500 years for Phobos and Deimos, while a back on the envelope calculation gives respectively 5400 and 1400 years for the precession of the node of these satellites submitted uniquely to Solar perturbation. The issue is then clear. By neglecting the torque exerted on the satellites orbits by the equatorial bulge of Mars, the authors found that instead of remaining close to the equatorial plane of Mars, as demonstrated by Goldreich (1965), the satellites were precessing along the orbital plane. Instead of finding a zero value for the mean precession torque (11), they obtain an effect following Eq. (10) which gives a contribution of about $0.5^{\prime \prime} /$ year to the precession of Mars axis, in agreement with the difference observed by the authors in Fig. 12. The authors should have wonder how Phobos and Deimos could both be at present with an inclination of less than 2 degrees with respect to the equator if they were precessing along the orbit. Goldreich indeed realized that this "would amount to an unbelievable coincidence".

\section{References}

Bouquillon, S., \& Souchay, J. 1999, A\&A, 345, 282

Edvardsson, S., Karlsson, K. G., \& Engholm, M. 2002, A\&A, 384, 689

Goldreich, P. 1965, AJ, 70, 5

Kinoshita, H. 1993, Celest. Mech. 57, 359

Murray, C. A. 1983, Vectorial Astrometry (Bristol: Adam Hilger Ltd) 